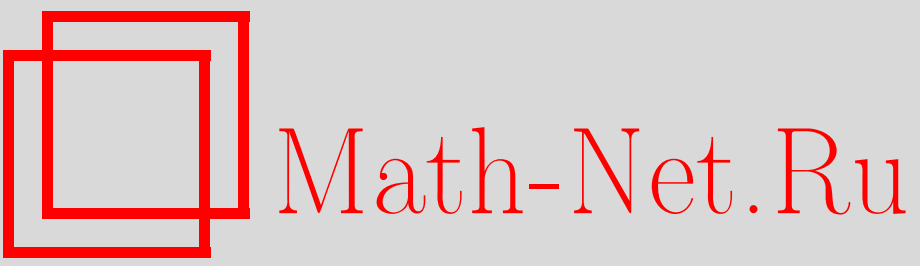

А. А. Давыдов, Х. Басто-Гонсалвеш, Управляемость неравенств в 2-особых точках, УМH, 2000, том 55, выпуск 6, 121-122

DOI: https://doi.org/10.4213/rm319

Использование Общероссийского математического портала Math-Net.Ru подразумевает, что вы прочитали и согласны с пользовательским соглашением

http://www.mathnet.ru/rus/agreement

Параметры загрузки:

IP: 54.237 .206 .68

26 апреля 2023 г., 13:10:50 


\title{
В МОСКОВСКОМ МАТЕМАТИЧЕСКОМ ОБЩЕСТВЕ
}

\author{
СООБШЕНИЯ МОСКОВСКОГО МАТЕМАТИЧЕСКОГО ОБШЕСТВА
}

\author{
УПРАВЛЯЕМОСТЬ НЕРАВЕНСТВ В 2-ОСОБЫХ ТОЧКАХ
}

\author{
А. А. ДАВЫДОВ, Х. БАСТО-ГОНСАЛВЕШ
}

\begin{abstract}
Динамическое неравенство задается гладкой функцией $F$ на пространстве скоростей в $\mathbb{R}^{n}$, $v$-допустимая скорость в точке $x$, если $F(x, v) \leqslant 0$. Множество $I(x)$ допустимых скоростей в точке $x$ называется индикатрисой скоростей этой точки. Здесь изучается случай локально ограниченных индикатрис. Типичное неравенство - точка некоторого открытого всюду плотного множества в пространстве соответствуюших функций $F$ в тонкой $C^{\infty}$-топологии.

Для неравенства $F$ допустимое движсние на интервале времени есть кусочно-дифференцируемое отображение $x$ этого интервала в фазовое пространство такое, что неравенство $F(x(t), \dot{x}(t)) \leqslant 0$ выполнено всюду на этом интервале. Точка $A$ достижима из точки $B$ за время $T$, если существует допустимое движение $x$ такое, что $x(0)=A$ и $x(T)=B$. Точка $A$ имеет свойство локальной транзитивности за малое время (=SLTP), если для любой ее окрестности $V$ и любого времени $T>0$ сушествует окрестность $V_{1}$ этой точки такая, что любые две точки из окрестности $V_{1}$ достижимы одна из другой за время $T$ без выхода из окрестности $V$. Точка $A$ обладает свойством локальной транзитивности (=LTP), если такая достижимость есть лишь для некоторого $T>0$.

Точка $A$ границы $S$ множества точек с SLTP называется $k$-особой, если существует проходящая через нуль опорная плоскость к индикатрисе $I(A)$, имеющая ровно $k$ общих точек с этой индикатрисой и лежащая в касательном множестве к $S$ в этой точке; $k \in \mathbb{N}$. При $n \geqslant 2$ типичное неравенство имеет $k$-особые точки лишь с $k \in\{1,2, \ldots, n\}$, и вблизи каждой из них $S$ есть гладко вложенная гиперповерхность в $\mathbb{R}^{n}$ (=разделяющая поверхность; см. [1] при $\left.k=1, n\right)$. При $n=2$ локальная управляемость типичных неравенств полностью изучена в [2].
\end{abstract}

Tеорема 1. При $n>2$ типичное неравенство имеет SLTP в любой $k$-особой точке при $n \geqslant k \geqslant 3$ и не имеет его в любой своей 1-особой точке.

Здесь наличие SLTP доказывается с помощью оценки "снизу" неравенства управляемой системой с нелучшими свойствами управляемости, но с SLTP в изучаемой точке, а отсутствие SLTP - с помощью оценки сверху неравенством с нехудшими свойствами управляемости, но без SLTP в этой точке. Итак, 2-особая точка - граничный случай наличия SLTP. Для типичного неравенства вблизи такой точки $P$ на разделяющей поверхности $S$ нулевая скорость - внутренняя точка "цилиндрической" части границы выпуклой оболочки $I(P)$, заполненной отрезками; структура

Работа выполнена при финансовой поддержке Российского фонда фундаменталњых исследований (грант № 00-01-00343), FCT и Galouste Gulbenkian Foundation. 
границы выпуклой оболочки типичной замкнутой поверхности описана в [3]. Такие отрезки, содержащие нулевую скорость, определяют на $S$ вблизи точки $P$ гладкое поле отрезков. Оно у типичного неравенства продолжается до гладкого поля $L$ в окрестности этой точки со значениями - направлениями отрезков из "цилиндрических" частей границ выпуклых оболочек индикатрис.

ТеОРема 2. Границы выпуклых оболочек индикатрис скоростей типичного неравенства задаются вблизи нулевой скорости в 2-особой точке Р равенством (возможно, после обращения и локальньх $C^{\infty}$-растяжений времени)

$$
\dot{z}=z-x^{2}+Y\left[A(x, y, z) \pm(E+\Lambda \dot{x})^{-1} Y^{*}\right]+\cdots,
$$

где $Y=\dot{y}-\left(z-x^{2}\right) \eta(x, y, z)$, “*” - знак транспонирования, $E$ - единичная матрица, $\eta, A$ - гладкие матричные функции, $\eta(P)=0, A(P)=0, \Lambda$ - диагональная матрица такая, что матрица $E+\Lambda \dot{x}$ положительно определена для всех $\dot{x}$ из пересечения $l(P)$ прямой $L(P)$ с выпуклой оболочкой индикатрисъ $I(P)$, в подходящей гладкой системе координат $x, y(\operatorname{dim} y=n-2), z$ с началом в точке $P$. При этом многоточие означает члень по крайней мере второго порядка по $Y$ u третьего по $Y u x, y, z$, а поле $L u$ разделяющая поверхность имеют форму $\mathbb{R} \frac{\partial}{\partial x}$ и $z=x^{2}$ соответственно.

Теорема 2 доказывается на основе того факта, что изучаемая часть границы выпуклой оболочки заполнена отрезками.

СледСтвиЕ 1. Типичное динамическое неравенство имеет SLTP в его 2-особой точке $P$, если в әтой точке интегральная кривая поля $L$ касается разделяющей поверхности изнутри множества точек, имеющих SLTP (=когда в равенстве (1) стоит знак "+").

Теорема 3. Если в 2-особой точке $P$ типичного неравенства в соответствующем равенстве (1) стоит знак "-", то әта точка имеет $S L T P$, если $A_{x}(P)^{*} A_{x}(P)>4, u$ не имеет LTP, если существует симметричная положительно определенная матрииа D размера $(n-2) \times(n-2)$ такая, что матрица

$$
\left(\begin{array}{cc}
4-A_{x}^{*} C A_{x} & -A_{x}^{*} C\left(A_{y}+D\right) \\
-\left(A_{x}^{*} C\left(A_{y}^{*}+D\right)\right)^{*} & 2 D-\left(D+A_{y}^{*}\right) C\left(D+A_{y}\right)
\end{array}\right)(P),
$$

где $C=E+\Lambda \dot{x}$, положительно определена для $\dot{x}$ на концах сегмента $l(P)$. При этом вблизи этой точки поверхность $2 z=-y D y^{*}$ является полупроницаемой для допустимых движений этого неравенства.

Первое утверждение этой теоремы доказывается с помощью подходящего выбора афффинной подсистемы из равенства (1) и использования известных результатов об управляемости систем; второе - прямой проверкой свойства полупроницаемости поверхности $2 z=-y D y^{*}$.

СлЕДСТвиЕ 2. При $n=3$ достаточнье условия отсутствия LTP в теореме 3 равносильны выполнению в точке $P$ двух неравенств: $(1+\lambda) A_{x}^{2}<4 u(1+\lambda)\left(8 A_{y}+A_{x}^{2}\right)<4$, əде $\lambda=\max \{\dot{x} \Lambda, \dot{x} \in l(P)\}$.

\section{СПИСОК ЛИТЕРАТУРЫ}

[1] Davydov A. A., Basto-Gonçalves J. // Chapman \& Hall/CRC Res. Notes Math. 2000. V. 412. Р. 223-235. [2] Давыдов А. А. // Труды МИАН. 1995. Т. 209. С. 84-123. [3] Закалюкин В. М. // Функц. анализ и его прил. 1977. Т. 11. № 3. С. 76-77. 